\title{
Reconstructing APT Datasets: Challenging the Limits of the Possible
}

\author{
François Vurpillot ${ }^{1}$, David Zanuttini ${ }^{1}$, Stefan Parviainen ${ }^{1}$, Baishaikhi Mazumder ${ }^{2}$, Nicolas Rolland ${ }^{1}$, \\ Constantinos Hatzoglou ${ }^{1}$ and James S. Speck ${ }^{3}$ \\ 1. Normandie Univ, UNIROUEN, INSA Rouen, CNRS, Groupe de Physique des Matériaux, Rouen, \\ France \\ 2. Department of Material Design and Innovation, University at Buffalo, Buffalo, NY, USA \\ 3. Materials Department, University of California, Santa Barbara, California, USA
}

Atom probe tomography (APT) has been successfully used in materials science for several decades for probing local compositional variations. In metals, the capacity to measure reliable composition at the nanoscale in 3D was successfully demonstrated in the early nineties [1, 2]. By using a few geometric ingredients, a reconstruction recipe was proposed at this time that surprisingly produced on selected materials, 3D images with an atomic scale precision of the distribution of atoms with elemental identification [3]. The ability to localize precisely the 3-D coordinates of individual atoms in direct space of materials is expected to find important applications in materials science and engineering, nanoscience, physics and chemistry. It is particularly crucial in semiconductor industry, to design optical devices or electronic devices, such as light-emitting diodes (LEDs), or transistors.

This ideal picture of near-perfect 3D microscope however faces two issues.

First, the precision of images may vary strongly from one material to another. Compared to an optical or an electron microscope, the spatial resolution loss is not diffraction limited, since image is produced from the inverse projection of ion trajectories, ions been produced by field evaporation of surface atoms. Precision is perturbed by slight deviations of the trajectories of ions from their initial positions at the specimen surface to the ion detector. A real understanding of this trajectory taking into account first the quantum interaction of atoms/ions with the specimen surface under the presence of the strong electric field existing on the surface is mandatory. The interplay between quantum processes, and more classical deviations induced by the distribution of the electric field close to the surface is extremely complex. In metals, most of the deviations thought to be induced by the field, as it was recently demonstrated experimentally by field ion microscopy in tungsten (fig.1). In oxides, quantum effects such as in-flight dissociation of molecules may degrade significantly the spatial precision (fig. 2) [4].

The second issue concerns the global distortions induced by the presence in the sample of phases very different in term of the critical fields required to extract surface atoms. This is a current case when analyzing devices composed of complex structures of metals, oxides and semiconductors materials. The surface of the sample ideally modelled with a constant curvature radius evolves dynamically under the process of field evaporation in a complex 3D surface. The direct consequence is the presence of distortions in the projected image. After the evaporation of each atom, the field distribution around the sample must be evaluated, to understand the projection law that should necessary be taken into account to generate an accurate reconstruction $[5,6]$. To overcome this cumbersome calculation, an alternative model, much simpler, was developed to reproduce the imaging process in simple configurations. In multilayers systems, it was demonstrated that most of the distortions could be reduced using a fast and efficient reconstruction algorithm that predicts the analytical shape of the emitting surface all along the evaporation process [7]. If a complementary technique can be used on the same sample to verify the morphology or provide complementary information before APT analysis, then this can also greatly 
facilitate the interpretation of the APT results. High angle annular dark field - scanning transmission electron microscopy (HAADF-STEM) imaging can be used for planar devices but for devices of a threedimensional nature, electron tomography (ET) becomes necessary. As the tip sample preparation requirements can be similar for both STEM/ET and APT and the spatial resolutions are comparable, combining electron tomography (ET) with APT on the same device becomes an attractive route to minimize APT artefacts and inaccuracies in device analysis. The complementary information from ET (in particular geometrical info) can be used to steer the reconstruction of the APT data (which has many adjustable parameters) in the right direction $[8,9][4]$.

\section{References:}

[1] A. Cerezo, TJ Godfrey, and GDW Smith, Review of Scientific Instruments, 59 (1988), p. 862.

[2] D. Blavette et al, Review of Scientific Instruments, 64 (1993), p. 2911.

[3] P. Bas et al, Appl Surf Sci. 87/88 (1995) p. 298.

[4] The authors acknowledge funding from the ANR / LABEX EMC3 / projects AQURATE and

DYNAMITE. Dr. Dagan from Oxford University is thanked for many useful discussions and contributions to this work.

[5] F Vurpillot, C Oberdorfer, Ultramicroscopy 159 (2015), p. 202.

[6] D. Beinke, C. Oberdorfer, and G. Schmitz Ultramicroscopy 465 (2016) p. 34.

[7] F Vurpillot et al, Semiconductor Science and Technology 31(2016) (7), 074002

[8] The authors acknowledge funding from the French ANR program (ANR-12-NANO-0001)

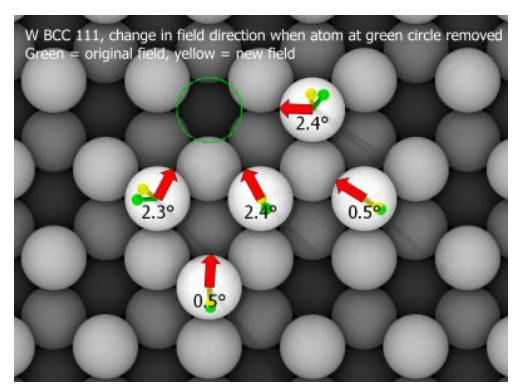

Figure 1. Molecular dynamic model on a tungsten field emitter surface used to calculate the apparent displacement (red arrows) of the surface atom positions in FIM, after the field evaporation of 1 atom from the surface (open circle). Field induced deviations are calculated to be predominant in this case.

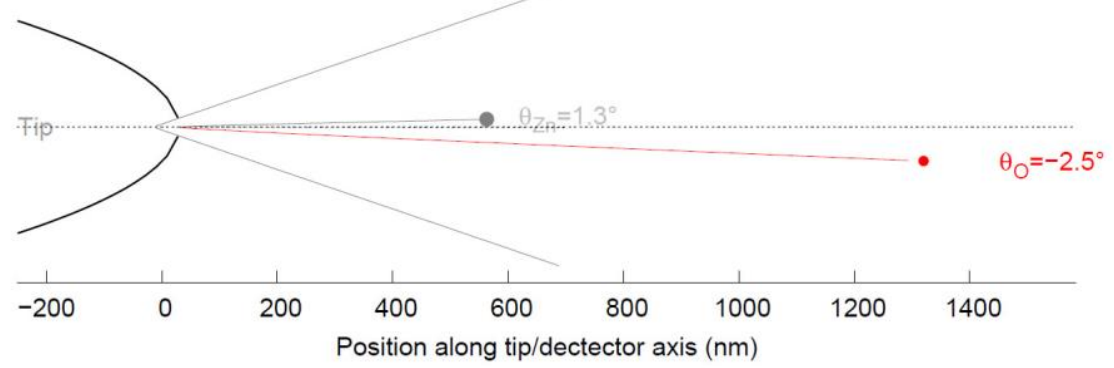

Figure 2. Calculated deviations of two ions $(\mathrm{O}+/ \mathrm{Zn}+)$ induced by the in-flight dissociation of the evaporated $\mathrm{ZnO} 2+$ molecule. $\mathrm{Ab}$ initio calculations were performed to calculate the dissociation probability, the dynamic of the emission process, and the impact on the spatial precision of the instrument. 